\title{
Use of ERCP in the United States over the past decade
}

\section{다 (요우}

Authors

Paul T. Kröner ${ }^{1}$, Mohammad Bilal², Ronald Samuel ${ }^{3}$, Shifa Umar ${ }^{4}$, Marwan S. Abougergi' ${ }^{5}$, Frank J. Lukens ${ }^{1}$, Massimo Raimondo ${ }^{1}$, David L. Carr-Locke ${ }^{6}$

Institutions

1 Division of Gastroenterology and Hepatology, Mayo Clinic, Jacksonville, Florida, United States

2 Division of Gastroenterology and Hepatology Beth Israel Deaconess Medical Center, Boston, Massachusetts, United State

3 Department of Internal Medicine, The University of Texas Medical Branch, Galveston, Texas, United States

4 Division of Gastroenterology, Allegheny Health Network, Pittsburgh, Pennsylvania, United States

5 Division of Gastroenterology, Palmetto Health, Columbia, South Carolina, United States

6 Division of Gastroenterology, New York-Presbyterian Hospital/Weill Cornell Medical Center, New York, New York, United States

submitted 26.9.2019

accepted after revision 12.2 .2020

Bibliography

DOI https://doi.org/10.1055/a-1134-4873 |

Endoscopy International Open 2020; 08: E761-E769

(c) Georg Thieme Verlag KG Stuttgart · New York

elSSN 2196-9736

Corresponding author

Paul T. Kröner, MD, MSc, Department of Gastroenterology and Hepatology, Mayo Clinic Florida, 4500 San Pablo Road South, Jacksonville, FL 32224, USA

Fax: +1-347-754-2702

thomaskroner@gmail.com

thomaskroner@ufm.edu

\section{ABSTRACT}

Background and study aims With newer imaging modalities, indications for use of endoscopic retrograde cholangiopancreatography (ERCP) have changed in the last decade. Despite advances in ERCP, paucity in recent literature regarding utilization and outcomes of ERCP exists. Thus, the aim of this study was to assess the inpatient use of $E R C P$, outcomes, and most common indications.

Patients and methods Retrospective-cohort study using the Nationwide Inpatient Sample 2007-2016. All patients with ICD9-10CM procedural codes for ERCP were included. The primary outcome was the use of ERCP. Secondary outcomes included determining procedural specifics (stenting, sphincterotomy and dilation), complications (post-ERCP pancreatitis [PEP], bile duct perforation), hospital length of stay, total hospital costs and charges. Multivariate regression analysis was used to adjust for confounders.

Results A total of 1,606,850 patients underwent inpatient ERCP. The mean age was 59 years ( $60 \%$ female). The total number of ERCPs increased over the last decade. Patients undergoing ERCP in 2016 had greater odds of undergoing bile duct stent placement, pancreatic duct (PD) stenting, biliary dilation, pancreatic sphincterotomy, PEP and biliary perforation. Inpatient mortality decreased. Hospital charges increased, while length of stay (LOS) decreased.

Conclusions The number of ERCPs increased in the past decade. Odds of therapeutic interventions and complications increased. The most common principal diagnoses were choledocholithiasis and gallstone-related AP. Hence, physicians must be aware to promptly diagnose and treat complications. These findings may reflect the increased case complexity and fact that ERCP continues to evolve into an increasingly interventional tool, contrasting from its former role as a predominantly diagnostic and gallstone extraction tool.

\section{Introduction}

Endoscopic retrograde cholangiopancreatography (ERCP) is a complex endoscopic procedure used to evaluate pancreatobiliary ductal anatomy through the use of fluoroscopy and contrast to determine suitable interventions for both benign and malignant pathology. ERCP was initially developed in the late 1960's as a diagnostic procedure and subsequently evolved to a therapeutic procedure with the first biliary sphincterotomy performed in 1974 in Kyoto, Japan [1-4]. In recent years, the role of ERCP has shifted from being a diagnostic and therapeutic procedure, to mainly being a therapeutic procedure [5]. Previous reports have shown that inpatient utilization of ERCP has been decreasing since 1996, which is believed to be due to the 
widespread availability of newer imaging modalities such as magnetic resonance cholangiopancreatography (MRCP) and endoscopic ultrasound (EUS) [5-8].

In the United States, current training in ERCP requires a prior 3-year training in internal medicine followed by a 3-year training program in general gastroenterology and finally an additional year in advanced endoscopy training. During that final year, the trainee is expected to acquire the necessary cognitive skills for decision-making, as well as the core ERCP technical skills $[9,10]$. As the demand for advanced endoscopists continues to increase, the number of academic programs offering ERCP training has increased, which highlights the importance of competency assessment in the area $[10,11]$. Some of the key factors that have been noted to influence competency positively include training at large-volume centers and a bile duct cannulation rate of $80 \%$ to $90 \%$, emphasizing that the total number of procedures to achieve proficiency varies among trainees and should, as such, be individualized [12-16].

Although previous epidemiological data are available on the topic, there have been significant recent technological and scientific advances in advanced endoscopy. There continues to be a paucity of recent large studies exploring temporal trends in the use of ERCP. Thus, the aim of the current study was to assess the current temporal trends in inpatient utilization of $E R C P$, and identification of common indications and interventions, procedural complications, and healthcare resource utilization using the largest public inpatient database in the United States throughout the past decade.

\section{Patients and methods}

\section{Study design and data source}

Patients were selected from the Nationwide Inpatient Sample (NIS) which is the largest publicly available, inpatient, all-payer database in the United States. The dataset for the years 2007 to 2016 contains more than 70 million hospital stays, which are a $20 \%$ stratified sample of over 4,000 non-federal acute care hospitals of more than 40 states of the United States, and is representative of $95 \%$ of hospital discharges nationwide. A principal diagnosis, defined as the primary discharge diagnosis, as well as up to 29 other secondary diagnoses are included in the dataset. The dataset also includes codes for up to 15 procedures performed during the hospital stay. It also allows determining length of hospital stay, and total hospitalization charges, as well as desired outcome measures such as calculations of inpatient disease prevalence. All analyzed data was extracted from the databases for the years 2007 to 2016 to design this retrospective cohort study.

\section{Study population}

All patients in the NIS dataset for 2007 to 2016 with an International Classification of Diseases, Ninth and Tenth Revisions, Clinical Modification (ICD-9 and ICD-10 CM) principal procedural code for ERCP were identified, signifying that only all patients undergoing inpatient ERCP were included. All codes are included in Supplemental Table 1. In all patients with an associated procedural code for ERCP, procedural specifics were obtained using their respective procedural ICD-9/10 codes. These included biliary sphincterotomy, biliary stenting, biliary dilatation, pancreatic sphincterotomy, PD stenting and PD dilatation. In addition, to evaluate the top indications for the procedures, the most common principal diagnostic codes were obtained from all patients with an associated procedural code for ERCP.

\section{Variable definition}

Patient general characteristics included demographics such as age, gender, ethnicity, median income in zip code, and insurance type. Hospital characteristics included hospital region, teaching status, number of hospital beds and hospital location. The HCUP divides the United States into four geographical locations into census regions: Northeast, Midwest, South and West. Each patient's vital status at the conclusion of hospital stay, total days of hospitalization and total hospitalization charges were also abstracted from the database. To account for patient comorbidities, the Deyo adaptation of the Charlson Comorbidity Index was used, which is a validated tool for large database analysis [17]. For ICD-9codes, the variable acute post-ERCP pancreatitis was defined using acute pancreatitis as a secondary diagnosis only if it was associated with an ERCP procedural code, and included only if the ERCP was not done on the day of the admission.

\section{Outcomes}

The aim of this study was to determine the temporal trends in the use of inpatient ERCP in the United States in the past decade.

Secondary outcomes were to determine the top five principal diagnoses associated with an associated procedural code for ERCP (hereafter referred to as ERCP indications), as well as comparing the ERCP indications in the year 2016 to the year 2007. In evaluating procedural complications, temporal trends in occurrence of ERCP-related perforation and post-ERCP pancreatitis (PEP) were examined. Temporal trends in resource utilization as measured by hospital costs, total hospitalization charges and length of stay were also explored.

\section{Statistical analysis}

Discharge-level weights published by the HCUP were used to estimate the total number of patients undergoing ERCP. Fisher's exact test was used to compare proportions and analysis of variance was utilized to compare means. To assess associations between ERCP and the outcomes of interest, adjusted odds ratios and adjusted means comparing the year 2016 to the year 2007 were obtained by multivariate logistic regression. The multivariate logistic regression model was constructed by first examining variables that are known to be associated with the outcomes on previous studies and conducting a univariate regression analysis. If the variables were associated with the outcomes on univariate analysis with a $P<0.10$, they were included in the multivariate model. Variables that were included in the model were age, gender, ethnicity, insurance carrier, median income in patient zip code, Charlson Comorbidity Index, weekend admission, hospital region, urban location, teaching 
status and number of hospital beds. All statistical analyses were conducted using STATA, Version 14 (StataCorp LP, College Station, Texas, United States).

\section{Results}

\section{Patient characteristics}

A total number of $1,606,850$ patients were identified who underwent inpatient ERCPs. Mean age was 59 years, and $60 \%$ were female. Baseline characteristics of patients who underwent ERCP in 2007 and 2016 are summarized in > Table 1. The majority of patients were white, followed by Hispanics. There was no significant difference in patient income or hospital region. As compared to 2007, patients had higher Charlson comorbidity index in 2016 (Charslon comorbidity index of $\geq 3$ : $28.2 \%$ vs $17.5 \%, P<0.01)$. More patients had Medicare $(48.1 \%$ vs $44.5 \%$ ) and Medicaid (15.2\% vs $11.4 \%$ ) insurance in 2016 as compared to 2007 . More patients were admitted to teaching hospitals in 2016 as compared to 2007 (73.0\% vs $47.4 \%$, respectively. $P<0.01)$.

\section{ERCP prevalence and indications}

The inpatient use of ERCP increased from $0.38 \%(148,179)$ in 2007 to $0.44 \%(156,874)$ in 2016 , (trend $P$ value $<0.01)$ ( $\triangleright$ Table 2, - Fig. 1).

The top five principal diagnosis for ERCP were: choledocholithiasis $(499,339,31.1 \%)$, acute biliary pancreatitis $(175,476$, $11.0 \%)$, ascending cholangitis $(79,607,4.9 \%)$, unspecified choledochal obstruction $(51,902,3.2 \%)$, and pancreatic head mass (37,223, 2.3\%).

From 2007 to 2016, the proportion of choledocholithiasis as the primary indication for ERCP decreased from $40.4 \%$ to $30.2 \%$ but it continued to be the primary indication for ERCP. The proportion of ERCP for acute cholangitis increased from $4.1 \%$ in 2007 to $10.8 \%$ in 2016 . Rates of ERCP for acute biliary pancreatitis decreased from $13.9 \%$ to $8.8 \%$, and for unspecified obstruction increased from $5.8 \%$ to $7.8 \%$, from 2007 to 2016 , respectively ( $\triangleright$ Table $\mathbf{3}$ ).

\section{ERCP-related interventions}

The rates of various ERCP related interventions are summarized in $>$ Table 4. Biliary sphincterotomy was the most common intervention performed (2007: 68.2\%, 2016: 62.6\%), followed by placement of biliary stents (2007: $23.6 \%$, 2016: $43.6 \%$ ). Biliary dilation was performed in $5.3 \%$ of cases in 2007 as compared to $10 \%$ in 2016 . PD stent placement was done in $9 \%$ of ERCPs in 2016 as compared to $5.1 \%$ in 2007.

After performing the multivariable analysis outlined in the methods section and adjusting for confounders, the use of biliary sphincterotomy (aOR: $0.94, P=0.12$ ), pancreatic sphincterotomy (aOR: 1.45, $P=0.06$ ), and PD dilation (aOR: $1.2, P=0.6$ ) was unchanged in 2016 compared to 2007 . On the other hand, the use of biliary stents (aOR: $2.17, P<0.01$ ), biliary dilation (aOR: 1.71, $P<0.01$ ), and PD stent placement (aOR: 1.57, $P<$ 0.01 ) significantly increased from 2007 to 2016.
- Table 1 Patient characteristics in 2007 and 2016.

\begin{tabular}{|l|c|c|c|}
\hline Variable & $\mathbf{2 0 0 7}$ & $\mathbf{2 0 1 6}$ & P value \\
\hline Age & 58.6 years & 60.3 years & $<0.01$ \\
\hline Female gender & $50.8 \%$ & $49.2 \%$ & $<0.01$ \\
\hline Ethnicity & & & \\
\hline - Caucasian & $67.2 \%$ & $67.1 \%$ & \\
\hline - African American & $8.6 \%$ & $9.1 \%$ & \\
\hline - Hispanic & $16.7 \%$ & $15.6 \%$ & 0.80 \\
\hline - Asian & $3.7 \%$ & $3.9 \%$ & \\
\hline - Other & & & \\
\hline
\end{tabular}

Other

Median Income in zip code

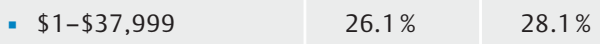

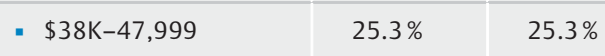

0.29

- $\$ 48 K-63,999$

\begin{tabular}{l|l}
$24.8 \%$ & $24.9 \%$
\end{tabular}

- $>\$ 64,000$

$23.9 \% \quad 21.7 \%$

Charlson Comorbidity Index

\begin{tabular}{l|l|l|}
\hline & $50.9 \%$ & $38.2 \%$ \\
\hline
\end{tabular}

\begin{tabular}{|l|l|l|l|}
\hline & $20.0 \%$ & $20.3 \%$ & $<0.01$ \\
\hline
\end{tabular}

\begin{tabular}{l|l|l|}
\hline & $11.6 \%$ & $13.2 \%$ \\
\hline
\end{tabular}

\begin{tabular}{|l|l|l}
\hline - 3 or more & $17.5 \%$ & $28.2 \%$ \\
\hline
\end{tabular}

Insurance

- Medicare

\begin{tabular}{l|l|}
\hline $44.5 \%$ & $48.1 \%$
\end{tabular}

- Medicaid

- Private

\begin{tabular}{|l|l|}
\hline $11.4 \%$ & $15.2 \%$ \\
\hline
\end{tabular}

$<0.01$

- Out of pocket

\begin{tabular}{l|l}
$34.3 \%$ & $29.2 \%$
\end{tabular}

- Other

$6.1 \%$

$3.7 \%$

$4.8 \%$

Hospital region

\begin{tabular}{|l|l|l|l|}
\hline - North & $20.8 \%$ & $19.0 \%$ & \\
\hline - Midwest & $21.6 \%$ & $21.9 \%$ & 0.56 \\
\hline - South & $34.1 \%$ & $36.2 \%$ & \\
\hline - West & $23.5 \%$ & $22.9 \%$ & \\
\hline Urban location & $92.7 \%$ & $96.6 \%$ & $<0.01$ \\
\hline Teaching hospital & $47.4 \%$ & $73.0 \%$ & $<0.01$ \\
\hline
\end{tabular}

\section{Bed size}

- Small
- Medium
- Large

Weekend admission

$9.1 \%$

$14.1 \%$

$24.9 \%$

$<0.01$

Table of baseline characteristics comparing patients undergoing ERCP in 2016 to 2007. 
> Table 2 Use of ERCP from 2007 to 2016.

\begin{tabular}{|c|c|c|c|c|c|c|c|c|c|c|}
\hline & 2007 & 2008 & 2009 & 2010 & 2011 & 2012 & 2013 & 2014 & 2015 & 2016 \\
\hline Total & 148,179 & 162,609 & 162,419 & 167,943 & 170,931 & 161,685 & 160,714 & 160,100 & 155,695 & 156,874 \\
\hline Percentage & $0.38 \%$ & $0.41 \%$ & $0.41 \%$ & $0.43 \%$ & $0.44 \%$ & $0.44 \%$ & $0.45 \%$ & $0.45 \%$ & $0.43 \%$ & $0.44 \%$ \\
\hline
\end{tabular}

Temporal trend in use of ERCP from 2007 to 2016. The total number of patients is shown, as well as the respective percentage relative to the total number of discharges each year.

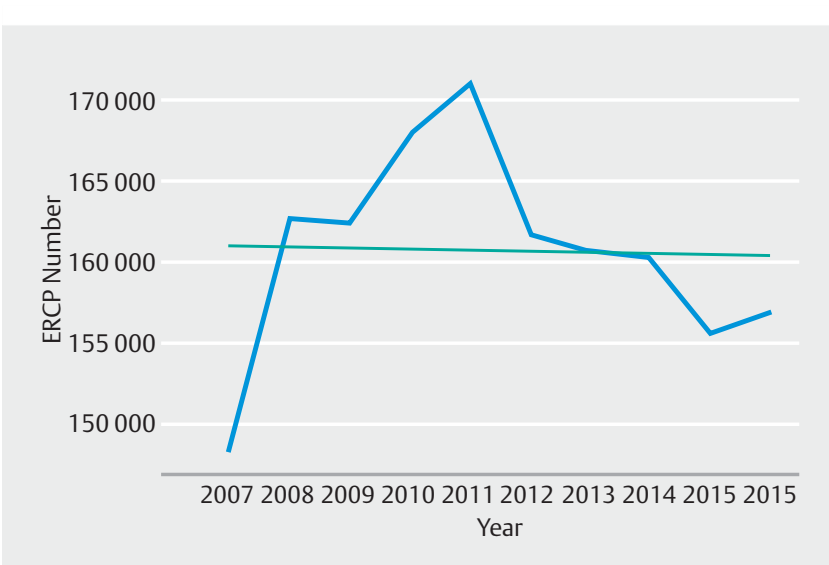

- Fig. 1 Graphic representation of temporal trends in the use of ERCP from 2007 to 2016.

> Table 3 Most common ERCP indications in 2016 compared to 2007.

\begin{tabular}{|c|c|c|c|}
\hline Indication & 2007 & 2016 & Percent change \\
\hline $\begin{array}{l}\text { Choledocholithiasis } \\
(\mathrm{N}=499,339)\end{array}$ & $40.4 \%$ & $30.2 \%$ & $-10.2 \%$ \\
\hline $\begin{array}{l}\text { Acute biliary pancreatitis } \\
(\mathrm{N}=175,476)\end{array}$ & $13.9 \%$ & $8.8 \%$ & $-5.1 \%$ \\
\hline $\begin{array}{l}\text { Ascending cholangitis } \\
(\mathrm{N}=79,607)\end{array}$ & $4.1 \%$ & $10.8 \%$ & $+6.7 \%$ \\
\hline $\begin{array}{l}\text { Unspecified obstruction } \\
(N=51,902)\end{array}$ & $5.8 \%$ & $7.8 \%$ & $+2.0 \%$ \\
\hline $\begin{array}{l}\text { Pancreatic head mass } \\
(\mathrm{N}=37,223)\end{array}$ & $2.5 \%$ & $2.9 \%$ & $+0.4 \%$ \\
\hline $\begin{array}{l}\text { Non-codable indication } \\
(\mathrm{N}=495,810)\end{array}$ & $33.3 \%$ & $39.5 \%$ & $+6.2 \%$ \\
\hline \multicolumn{4}{|c|}{$\begin{array}{l}\text { Top principal diagnoses associated with procedural codes of ERCP in } 2016 \\
\text { compared to 2007. Although the most common indications were similar, use } \\
\text { of ERCP for choledocholithiasis and acute biliary pancreatitis decreased, } \\
\text { while use of ERCP for ascending cholangitis and non-codable indications in- } \\
\text { creased. }\end{array}$} \\
\hline
\end{tabular}

\section{Timing to ERCP}

The mean time to ERCP in patients with acute cholangitis as well as acute biliary pancreatitis decreased from 2.6 days in 2007 to 2.2 days in $2016(P<0.01)$.

After adjusting for confounders, the mean time to ERCP in patients with acute cholangitis [adjusted mean time difference:
-0.7 days ( $95 \% \mathrm{Cl}:-0.9$ to $-0.4, P<0.01$ ) as well as acute biliary pancreatitis (adjusted mean time difference: -0.7 days [ $95 \% \mathrm{Cl}$ : -0.9 to $-0.6, P<0.01]$ ) decreased in 2016 as compared to 2007.

\section{ERCP-related complications}

The percentage of post-ERCP pancreatitis (PEP) increased from $12.3 \%$ to $16.5 \%$ from 2007 to 2016. After adjusting for confounding factors, the odds of PEP were significantly higher in 2016 as compared to 2007 (aOR: 1.49, 95\% Cl: 1.39-1.60, $P<$ 0.01 ( $\triangleright$ Table 5).

The rates of ERCP-related perforation remained low over the past decade (2007: $0.1 \%, 2016: 0.3 \%$ ), but significantly increased in 2016 as compared to 2007 (aOR: 3.65, 95\% Cl: 1.6 to $7.9, P<0.01)$.

\section{Resource utilization}

The mean length of stay (LOS) for patients who underwent ERCP was 7 and 6.1 days in 2007 and 2016, respectively. After adjusting for confounding factors, the LOS was significantly decreased in 2016 as compared to 2007 (adjusted mean difference in LOS: -1.6 Days, $P<0.01,95 \% \mathrm{Cl}:-1.9$ to $-1.4, P<0.01$ ).

The mean total hospitalization charges increased from $\$ 56,577$ in 2007 to $\$ 77,804$ in 2016 . This difference persisted after adjusting for confounding factors with mean additional total hospitalization charges of \$14,729 in 2016 as compared to 2007 (95\% Cl: $\$ 10,218$ to $\$ 19,239$ ), $P<0.01$ ) ( Table 6).

\section{Discussion}

In the current study, we used the largest publically available inpatient database to show that the annual inpatient utilization of ERCP continues to increase over the past decade. Even though choledocholithiasis continues to remain the most common indication for ERCP, the proportion of ERCPs for choledocholithiasis decreased from $40.4 \%$ to $30.2 \%$ over the past 10 years. We also noted that there has been shift in the utilization rates of ERCP to other interventional indications. An increased proportion of ERCPs are now being done for cholangitis, while the use of ERCP in acute biliary pancreatitis is decreasing. In addition, we show that while rates of biliary sphincterotomy, pancreatic sphincterotomy and PD dilation remain constant over the past decade, while the use of biliary stents, pancreatic stents and biliary dilation significantly increased over this time period. In regards to ERCP-related complications, rates of postERCP pancreatitis were noted to have increased in the past decade despite the current emphasis on preventive measures such as double wire technique, rectal indomethacin and PD stenting. 
- Table4 Intra-procedural interventions in ERCP in 2016 compared to 2007.

\begin{tabular}{|l|l|l|l|l|}
\hline Procedural Specific & $\mathbf{2 0 0 7}$ & $\mathbf{2 0 1 6}$ & $\mathbf{P}$ value & aOR (95\% Cl), $\boldsymbol{P}$ value \\
\hline Biliary sphincterotomy & $101,097(68.2 \%)$ & $98,250(62.6 \%)$ & $<0.01$ & $\mathbf{0 . 9 4}(\mathbf{0 . 8 6 - 1 . 0 2 ) , 0 . 1 2}$ \\
\hline Biliary dilatation & $7,889(5.3 \%)$ & $15,745(10.0 \%)$ & $<0.01$ & $\mathbf{1 . 7 1}(1.41-2.07),<0.01$ \\
\hline Biliary stenting & $35,013(23.6 \%)$ & $68,365(43.6 \%)$ & $<0.01$ & $\mathbf{2 . 1 7}(1.37-1.68),<0.01$ \\
\hline Pancreatic sphincterotomy & $302(0.2 \%)$ & $480(0.3 \%)$ & $<0.01$ & $1.45(0.99-2.13), 0.06$ \\
\hline Pancreatic duct dilatation & $607(0.4 \%)$ & $975(0.6 \%)$ & 0.05 & $1.20(0.62-2.32), 0.59$ \\
\hline Pancreatic duct stenting & $7,503(5.1 \%)$ & $13,975(9.0 \%)$ & $<0.01$ & $1.57(1.28-1.91),<0.01$ \\
\hline $\begin{array}{l}\text { Intra-procedural specifics in patients undergoing ERCP in } 2016 \text { compared to } \\
\text { ported. }\end{array}$ & & & \\
\hline
\end{tabular}

- Table 5 Occurrence of Post-ERCP pancreatitis from 2007 to 2016

\begin{tabular}{|c|c|c|c|c|c|c|c|c|c|c|c|}
\hline Year & 2007 & 2008 & 2009 & 2010 & 2011 & 2012 & 2013 & 2014 & 2015 & 2016 & Total \\
\hline $\begin{array}{l}\text { Total (per- } \\
\text { centage) }\end{array}$ & $\begin{array}{l}18,161 \\
(12.3 \%)\end{array}$ & $\begin{array}{l}21,623 \\
(13.3 \%)\end{array}$ & $\begin{array}{l}21,326 \\
(13.3 \%)\end{array}$ & $\begin{array}{l}21,824 \\
(13.0 \%)\end{array}$ & $\begin{array}{l}22,615 \\
(13.2 \%)\end{array}$ & $\begin{array}{l}21,250 \\
(13.1 \%)\end{array}$ & $\begin{array}{l}21,720 \\
(13.5 \%)\end{array}$ & $\begin{array}{l}21,590 \\
(13.5 \%)\end{array}$ & $\begin{array}{l}22,910 \\
(14.7 \%)\end{array}$ & $\begin{array}{l}25,804 \\
(16.5 \%)\end{array}$ & $\begin{array}{l}218,812 \\
(13.6 \%)\end{array}$ \\
\hline
\end{tabular}

Adjusted $1.49(1.39-1.60),<0.01$

odds ratio

$(95 \% \mathrm{Cl})$,

$P$ value

Occurrence of post-ERCP pancreatitis in number and percentage of cases during the period from 2007 to 2016 as well as adjusted odds ratio, confidence interval and $P$ value for 2016 versus 2007.

D Table 6 Resource utilization associated with use of ERCP comparing 2007 to 2016.

\begin{tabular}{|l|l|l|l|l|}
\hline & \multicolumn{2}{|c|}{ Unadjusted } & \multicolumn{1}{l|}{ Adjusted } \\
\hline & $\mathbf{2 0 0 7}$ & $\mathbf{2 0 1 6}$ & $\mathbf{P}$ value & aOR $(\mathbf{9 5} \% \mathbf{C l}), \boldsymbol{P}$ value \\
\hline Hospital costs & $\$ 17,814$ & $\$ 18,782$ & $<0.01$ & $-\$ 865(-\$ 1,769-\$ 39), 0.06$ \\
\hline Hospitalization charges & $\$ 56,577$ & $\$ 77,804$ & 0.01 & $\$ 14,729(\$ 10,218-\$ 19,239),<0.01$ \\
\hline Hospital Length of Stay & 7.0 & 6.1 & 0.01 & $-1.6(-1.9,-1.4)<0.01$ \\
\hline
\end{tabular}

Economic burden of ERCP from 2007 to 2016. Unadjusted and adjusted costs, charges and length of hospital stay are presented.

ERCP-related perforations have also increased from 2007 to 2016. As far as resource utilization trend, despite length of hospital stay becoming shorter over the past 10 years, the total hospitalization charges increased over time.

Our study showed that there was increasing trend of inpatient utilization of ERCP over the past decade. Although a numerical difference was found, it is not significantly striking $(0.38$ $\%-0.44 \%$ ) in the study period. This finding is contrary to previous studies by Mazen and Ahmed which showed a decrease in inpatient utilization of ERCP $[5,6]$. In previous reports, the decreasing number of inpatient ERCP was thought to be due to two factors. First, since ERCP has now become a primarily therapeutic procedure rather than both diagnostic and therapeutic due to the availability of MRCP and EUS [6]. The second being the increasing outpatient utilization of ERCP [18]. Thus, the NIS would not detect these patients. Lastly, some of the previous studies did not include certain procedural ICD codes pertaining to ERCP. Despite this, our study shows that the inpatient use of ERCP has increased over the past decade. We believe that this finding is not necessarily surprising. Over the past decade, even though use of ERCP as a diagnostic modality has rightfully decreased, there have been significant strides in therapeutic ERCP. With the advent of EUS and MRCP, use of ERCP perhaps underwent a transient decline. However, use of cholangioscopy and ERCP for complex biliary stone and biliary pathology has been increasing and evolving. This is also consistent with the increasing number of training programs for advanced endoscopy fellowship, which results in an increased absolute number of physicians being able to perform ERCP, and then translated into increased total number of ERCPs performed [11]. These findings were also seen in another study by Moffatt et al. which included both inpatient and outpatient ERCPs from 1984 to 2009 and showed an overall increase in total number of procedures [19]. However, further studies are 
needed using both outpatient and inpatient populations to determine an accurate number of annual ERCPs in the United States.

Our study also showed that choledocholithiasis and acute biliary pancreatitis were by far the primary indications for inpatient ERCP, which is consistent with previous studies [5, 19,20]. However, we did note that there have been shifts in the proportions of indications for ERCP. Even though choledocholithiasis remains the most common indication for ERCP, the proportion of ERCP in patients with acute biliary pancreatitis has decreased. This is expected and consistent with other recent studies, since consensus guidelines recommend that ERCP should only be performed in acute biliary pancreatitis patients if they have cholangitis or have documented or increased suspicion of choledocholithiasis [21,22]. Interestingly, our analysis showed that a greater number of ERCPs are being performed for cholangitis now as compared to 10 years ago. This could be due to the increasing availability of ERCP. Another reason for this result could be that ICD-10-CM now has more specific codes for acute cholangitis as compared to ICD-9-CM.

Additionally, our study has shown that while the use of several standard ERCP-related interventions (i. e. biliary sphincterotomy, pancreatic sphincterotomy) has remained constant, the use of some ERCP-related interventions such as use of biliary stents, pancreatic duct (PD) stents and biliary dilation has increased at a statistically significant level over the past decade. There could be multiple reasons for this. Foremost, is that ERCP is now primarily a therapeutic procedure, so more patients are likely to undergo ERCP-related interventions. Another reason for this is likely related to the advancements in endoscopic techniques and accessories which has made ERCP more popular in the use of complex pancreatobiliary diseases. Also, much more widespread availability and options for biliary stents are also likely contributing to this. In regards to the increased use of PD stent placements over the past decade, this is likely due to overwhelming data showing reduction in PEP after PD stent placement [22]. On the other hand, increased use of PD stent placement could also be due to increased undertaking of ERCP for therapy of pancreatic diseases. Similarly, the increased utilization of biliary dilation is likely reflective of advances in modern technology and the increased use of ERCP in complex biliary pathology. Another plausible reason could be that, as liver transplantation continues to increase, need for ERCP in these patients has also increased [23]. In one study, the utilization of ERCP in liver transplant recipients was reported to be as high as $45 \%$ [24].

Our analysis showed an increasing trend of overall inpatient ERCP-related complications over time. The current inpatient rate of PEP is $16.5 \%$ and the odds of PEP have increased over time. The rate of PEP in our study is much higher than most previous studies in which it ranges around 4\% [25-28]. Even though Cheng et al. did a multicenter study of 1115 patients and reported a PEP rate of $15.1 \%$ [29], we feel that this number is likely an over representation since the ICD-CM does not have a specific code for PEP. In addition, the fact that only inpatient procedures are being evaluated in this study may also play a role. It is speculated that inpatients may have a higher comor- bidity burden and may be experiencing a clinical situation (which has them admitted in the hospital) that may be placing them at higher risks of complications. Despite this, the overall increasing trend of PEP is concerning. This could be related to increasing use of ERCP for complicated cases warranting hospital admission, as mentioned above, which is likely resulting in a greater number of cases of PEP. This is also evident by the fact that our analysis showed that the patients undergoing ERCP in 2016 tended to have a higher comorbidity burden (increased Charlson comorbity index), and that a greater number of patients are undergoing ERCP at teaching hospitals. Previously, one large systematic review has shown that center and endoscopists with low ERCP volume are more likely to have ERCPrelated complications. ERCP-related perforation has previously been noted to be 0.3 to $0.6 \%$ [30-33]. Our study showed similar rates of ERCP-related perforations. However, the odds of ERCP related perforations have also increased over the past decade. This again could be related to the increasing use of ERCP for more complex cases, but also raises the concern of lack of standardized ERCP training, as there continue to be an increasing number of advanced endoscopy fellowship training programs [11].

In regards to healthcare resource utilization, mean LOS for patients undergoing ERCP ranged from 7.0 to 6.1 days from 2007 to 2106, respectively. This is consistent with seen in other studies [5]. However, despite that the reasons for hospitalization in patients undergoing ERCP barely changed (gallstonerelated disease and ductal strictures in 2007 compared to gallstone-related disease, pancreatic head masses and ductal strictures in 2016), deeper knowledge on the baseline comorbidities between the two groups would have been key to better explore the differences in resource utilization. Despite the decrease in LOS, we found that total hospitalization charges increased over time, which is a trend observed across all medical conditions, and is assumed to be due to changing insurance policies and models.

There are some limitations to our study. First, it is a retrospective analysis due to the nature of the NIS database. Second, due to the administrative nature of the database, it has been documented that claims-based databases are susceptible to inaccurately entered or missing codes [34]. However, ICD-9 CM codes have been shown to have a high specificity and sensitivity when used to study gastrointestinal diseases [35]. Third, we were not able to identify the level of complexity of ERCP, prior ERCP history (index or repeat ERCP, native papilla), and use of preventative measures against PEP as these factors are not coded in the NIS. Moreover, the NIS only has data on inpatient data and thus, our study did not include outpatient ERCP utilization rates, which now constitute a large number of ERCPs. Also, the NIS does not contain information regarding use of medications and laboratory data. The ICD-9/10-CM coding system does not have a diagnostic code for PEP as well, which is perhaps contributing to the increased number of PEP reported in our cohort. Last, readmissions are unable to be tracked within the database.

Despite these limitations, our study has several strengths. Our study provides the latest and most up-to-date data on in- 
patient use of ERCP using the largest national inpatient database in the United States. We also used the newly re-designed NIS 2016 database which using the latest ICD-10-CM coding system which has more specific diagnostic codes for diseases such as acute biliary pancreatitis and cholangitis. This information was lacking in prior studies due to the use of ICD-9-CM coding system. Therefore, our study is the first evaluating the comprehensive information regarding ERCP using both ICD-9 as well as ICD-10 diagnostic codes. Our study also shows that after a transient decline in inpatient use of ERCP, the utilization of inpatient ERCP is increasing once again. This is accompanied by a speculated increased-complexity of ERCP cases as evident by increased use of ERCP-related interventions and post-ERCP complications. This information is especially important in the current era of increasing interventional ERCP. As the number of advanced endoscopy training programs increases and there continues to be a strong interest in additional advanced endoscopic procedures such as per-oral endoscopic myotomy (POEM), third space endoscopy, and endo-bariatric procedures, the focus of these training programs might shift away from the rigorous ERCP training of the past. Therefore, advanced endoscopy training programs should still focus on competence based ERCP training as outlined by the American Society of Gastrointestinal Endoscopy (ASGE) [36].

\section{Conclusion}

In conclusion, inpatient use of ERCP is increasing. This is accompanied by an increase in ERCP-related interventions likely reflective of an increasing complexity of cases. The rate of postERCP complications also is increasing. Further studies are needed to identify whether the complexity of ERCP procedures and related indications are changing, as well as determining whether the technique is associated with increased observed complications. More data on outpatient utilization of ERCP along with its associated resource utilization burden is also needed.

\section{Competing interests}

The authors declare that they have no conflict of interest.

\section{References}

[1] McCune WS, Shorb PE, Moscovitz H. Endoscopic cannulation of the ampulla of vater: a preliminary report. Annals of surgery 1968; 167 : 752-756

[2] Cotton PB. Cannulation of the papilla of Vater by endoscopy and retrograde cholangiopancreatography (ERCP). Gut 1972; 13: $1014-$ 1025

[3] Kawai K, Akasaka Y, Murakami K et al. Endoscopic sphincterotomy of the ampulla of Vater. Gastrointestinal endoscopy 1974; 20: 148-151

[4] Rabinov KR, Simon M. Peroral cannulation of the ampulla of Vater for direct cholangiography and pancreatography. Preliminary report of a new method. Radiology 1965; 85: 693-697

[5] Ahmed M, Kanotra R, Savani GT et al. Utilization trends in inpatient endoscopic retrograde cholangiopancreatography (ERCP): A crosssectional US experience. Endosc Int Open 2017; 5: E261-271
[6] Mazen JM, Yoon EJ, Saadi A et al. Trends in the utilization of endoscopic retrograde cholangiopancreatography (ERCP) in the United States. The American journal of gastroenterology 2007; 102: 966975

[7] Huang RJ, Thosani NC, Barakat MT et al. Evolution in the utilization of biliary interventions in the United States: results of a nationwide longitudinal study from 1998 to 2013. Gastrointestinal endoscopy 2017; 86: 319-326.e315

[8] Yachimski PS, Ross A. The Future of Endoscopic Retrograde Cholangiopancreatography. Gastroenterology 2017; 153: 338-344

[9] Walsh CM. In-training gastrointestinal endoscopy competency assessment tools: Types of tools, validation and impact. Best practice \& research. Clinical gastroenterology 2016; 30: 357-374

[10] Jorgensen J, Kubiliun N, Law JK et al. Endoscopic retrograde cholangiopancreatography (ERCP): core curriculum. Gastrointestinal endoscopy 2016; 83: 279-289

[11] Elta GH, Jorgensen J, Coyle WJ. Training in interventional endoscopy: current and future state. Gastroenterology 2015; 148: 488-490

[12] Cote GA, Imler TD, Xu H et al. Lower provider volume is associated with higher failure rates for endoscopic retrograde cholangiopancreatography. Medical care 2013; 51: 1040-1047

[13] Dave-Verma H, Moore S, Singh A et al. Computed tomographic enterography and enteroclysis: pearls and pitfalls. Curr Probl Diagn Radiol 2008; 37: 279-287

[14] Shahidi N, Ou G, Telford J et al. When trainees reach competency in performing ERCP: a systematic review. Gastrointestinal endoscopy 2015; 81: 1337-1342

[15] Eisen GM, Baron TH, Dominitz JA et al. Methods of granting hospital privileges to perform gastrointestinal endoscopy. Gastrointestinal endoscopy 2002; 55: 780-783

[16] Wani S, Keswani RN, Petersen B et al. Training in EUS and ERCP: standardizing methods to assess competence. Gastrointestinal endoscopy 2018; 87: 1371-1382

[17] Deyo RA, Cherkin DC, Ciol MA. Adapting a clinical comorbidity index for use with ICD-9-CM administrative databases. Journal of clinical epidemiology 1992; 45: 613-619

[18] Kozarek RA. The future of ERCP. Endosc Int Open 2017; 5: E272-E274

[19] Moffatt DC, Yu BN, Yie W et al. Trends in utilization of diagnostic and therapeutic ERCP and cholecystectomy over the past 25 years: a population-based study. Gastrointestinal endoscopy 2014; 79: 615622

[20] Coelho-Prabhu N, Shah ND, Van Houten H et al. Endoscopic retrograde cholangiopancreatography: utilisation and outcomes in a 10year population-based cohort. BMJ Open 2013: 3

[21] Bilal M, Kline KT, Trieu JA et al. Trends in same-admission cholecystectomy and endoscopic retrograde cholangiopancreatography for acute gallstone pancreatitis: A nationwide analysis across a decade. Pancreatology : official journal of the International Association of Pancreatology (IAP) 2019; 19: 524-530

[22] Buxbaum JL, Abbas Fehmi SM, Sultan S et al. ASGE guideline on the role of endoscopy in the evaluation and management of choledocholithiasis. Gastrointestinal endoscopy 2019; 89: 1075-1105.e1015

[23] Network OPaT. National Data.https://optn.transplant.hrsa.gov/data/ view-data-reports/national-data/\# [Accessed June 2, 2019]

[24] Barritt AS, Miller CB, Hayashi PH et al. Effect of ERCP utilization and biliary complications on post-liver-transplantation mortality and graft survival. Digestive diseases and sciences 2010; 55: 3602-3609

[25] Cheon YK, Cho KB, Watkins JL et al. Frequency and severity of postERCP pancreatitis correlated with extent of pancreatic ductal opacification. Gastrointestinal endoscopy 2007; 65: 385-393

[26] Freeman ML, DiSario JA, Nelson DB et al. Risk factors for post-ERCP pancreatitis: a prospective, multicenter study. Gastrointestinal endoscopy 2001; 54: 425-434 
[27] Wang P, Li ZS, Liu F et al. Risk factors for ERCP-related complications: a prospective multicenter study. The American journal of gastroenterology 2009; 104: 31-40

[28] Rabenstein T, Schneider HT, Bulling D et al. Analysis of the risk factors associated with endoscopic sphincterotomy techniques: preliminary results of a prospective study, with emphasis on the reduced risk of acute pancreatitis with low-dose anticoagulation treatment. Endoscopy 2000; 32: 10-19

[29] Cheng CL, Sherman S, Watkins JL et al. Risk factors for post-ERCP pancreatitis: a prospective multicenter study. The American journal of gastroenterology 2006; 101: 139-147

[30] Freeman ML. Complications of endoscopic biliary sphincterotomy: a review. Endoscopy 1997; 29: 288-297

[31] Loperfido S, Angelini G, Benedetti G et al. Major early complications from diagnostic and therapeutic ERCP: a prospective multicenter study. Gastrointestinal endoscopy 1998; 48: 1-10
[32] Masci E, Toti G, Mariani A et al. Complications of diagnostic and therapeutic ERCP: a prospective multicenter study. The American journal of gastroenterology 2001; 96: 417-423

[33] Howard T], Tan T, Lehman GA et al. Classification and management of perforations complicating endoscopic sphincterotomy. Surgery 1999; 126: 658-663; discussion 664-655

[34] Klabunde CN, Warren JL, Legler JM. Assessing comorbidity using claims data: an overview. Medical care 2002; 40: Iv-26-35

[35] Cooper GS, Chak A, Lloyd LE et al. The accuracy of diagnosis and procedural codes for patients with upper GI hemorrhage. Gastrointestinal endoscopy 2000; 51: 423-426

[36] American Society for Gastrointestinal Endoscopy. Quality indicators for ERCP.https://www.asge.org/docs/default-source/education/practice_guidelines/doc-427772e8-226f-4d33-96b3-cd26f1ce95ff.pdf? sfvrsn=6 [Accessed June 2, 2019] 
- Supplemental Table 1 Utilized ICD9/10 diagnostic and procedural codes. ICD-9 and ICD-10 diagnostic and procedural codes used

\begin{tabular}{|c|c|}
\hline Diagnosis/procedure & ICD-9/ ICD-10 codes \\
\hline $\begin{array}{l}\text { Endoscopic retrograde } \\
\text { cholangiopancreatography }\end{array}$ & $\begin{array}{l}\text { 51.10, 51.11, 51.84, 51.85, 51.88, 52.13, 52.14, 52.93, 0FJB8ZZ, 0FJD8ZZ, 0F9C8ZZ, 0F758DZ, } \\
\text { 0F768DZ, 0F788DZ, 0F798DZ, 0F7C8DZ, 0F9580Z, 0F9680Z, 0F9880Z, 0F9980Z, 0F9C80Z, } \\
\text { 0FC58ZZ, 0FC68ZZ, 0FC88ZZ, 0FC98ZZ, 0FCC8ZZ, 0FF58ZZ, 0FF68ZZ, 0FF88ZZ, 0FF98ZZZ, 0FFC8ZZ, } \\
\text { 0FJD8ZZ, 0F7D8DZ, 0F7F8DZ, 0F9D80Z, 0F9F80Z, 0FCD8ZZ, 0FCF8ZZ, 0FFD8ZZ, 0FFF8ZZ }\end{array}$ \\
\hline Biliary sphincterotomy & $\begin{array}{l}\text { 51.85, 0F997ZX, 0F998ZX, 0F9C7ZX, 0F9C8ZX, 0F5C8ZZ, 0FCC8ZZ, 0FFC8ZZ, 0FNC8ZZ, 0FNC8Z, } \\
\text { 0FNC8, 0F5C8ZZ, 0F7C8ZZ, 0F7C8DZ, 0F9C80Z, 0F9C8ZX, 0F9C8ZZ, 0F9C40Z, 0F9C4ZX, 0F9C4ZZ, } \\
\text { 0FCC8ZZ, 0FCC4ZZ, 0FC98ZZ, 0FDC8ZX, 0FD98ZX, 0F9980Z, 0F998ZX, 0F998ZZ, 0FFC8ZZ, } \\
\text { 0FNC8ZZ, 0FBC8ZX, 0FBC8ZZ }\end{array}$ \\
\hline Biliary ductal dilation & 51.84, OF758ZZ, 0F768ZZ, 0F788ZZ, 0F798ZZ \\
\hline Biliary duct stenting & $\begin{array}{l}\text { 51.87, 0F758DZ, 0F768DZ, 0F788DZ, 0F798DZ, 0F7C8DZ, 0F9580Z, 0F9680Z, 0F9880Z, 0F9980Z, } \\
\text { 0F9C80Z }\end{array}$ \\
\hline Pancreatic sphincterotomy & $51.82,0$ F9D8ZX, 0F9D8ZZ \\
\hline Pancreatic ductal dilation & 52.98, 0F7D8ZZ, 0F7F8ZZ \\
\hline Pancreatic duct stenting & $52.93,0 F 7 D 8 D Z, 0 F 7 F 8 D Z, 0 F 9 D 80 Z, 0 F 9 F 80 Z$ \\
\hline Ampullectomy & $51.64,0 F 5 C 8 Z Z, 0 F B C 8 Z Z$ \\
\hline Bile duct perforation & $576.3, \mathrm{~K} 83.2, \mathrm{~K} 83.3$ \\
\hline $\begin{array}{l}\text { Post-procedural bleeding (with associated } \\
\text { ERCP procedure codes) }\end{array}$ & $998.1,998.11,998.12,998.13$, K91.84, K91.840, K91.841 \\
\hline Cholangitis & $576.1, \mathrm{~K} 83.0, \mathrm{~K} 83.08$ \\
\hline Biliary acute pancreatitis & K85.10, K85.11, K85.12, K85.1 \\
\hline
\end{tabular}

